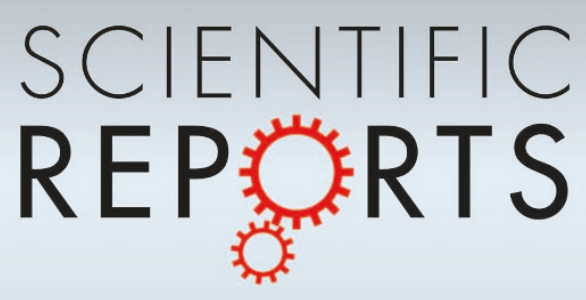

OPEN

SUBJECT AREAS:

TOPOLOGICAL

INSULATORS

ELECTRONIC PROPERTIES AND MATERIALS

ELECTRONIC DEVICES

ELECTRONIC STRUCTURE

Received

2 November 2012

Accepted

2 April 2013

Published

11 April 2013

Correspondence and requests for materials should be addressed to H.J. (h-jin@

northwestern.edu)

* These authors contributed equally to this work.

\section{Topological Oxide Insulator in Cubic Perovskite Structure}

\author{
Hosub Jin*, Sonny H. Rhim*, Jino Im* \& Arthur J. Freeman \\ Department of Physics and Astronomy, Northwestern University, Evanston, Illinois 60208, USA.
}

The emergence of topologically protected conducting states with the chiral spin texture is the most prominent feature at the surface of topological insulators. On the application side, large band gap and high resistivity to distinguish surface from bulk degrees of freedom should be guaranteed for the full usage of the surface states. Here, we suggest that the oxide cubic perovskite $\mathrm{YBiO}_{3}$, more than just an oxide, defines itself as a new three-dimensional topological insulator exhibiting both a large bulk band gap and a high resistivity. Based on first-principles calculations varying the spin-orbit coupling strength, the non-trivial band topology of $\mathrm{YBiO}_{3}$ is investigated, where the spin-orbit coupling of the $\mathrm{Bi} 6 p$ orbital plays a crucial role. Taking the exquisite synthesis techniques in oxide electronics into account, $\mathrm{YBiO}_{3}$ can also be used to provide various interface configurations hosting exotic topological phenomena combined with other quantum phases.

ollowing the great success of semiconductors, oxide electronics have recently drawn a lot of attention ${ }^{1,2}$. The main reason for this growing attraction might be attributed to the versatile ground states of oxide systems that show various type of order parameters ${ }^{3}$. Moreover, heterostructures of several different oxide perovskites realize artificial composite systems with integrated functionality, and accelerate the growth of this field ${ }^{4,5}$. Introducing a new quantum phase to oxide systems, therefore, has much influence in enriching and developing new physics. In this context, finding an oxide system with non-trivial band topology, so-called topological insulator $(\mathrm{TI})^{6,7}$, and possibly combining oxide electronics with TI is one of the most intriguing and significant tasks.

However, due to the small spin-orbit coupling (SOC) strength of oxygen atoms, the TI phase is not easily accessible in oxides compared to the heavy chalcogenides ${ }^{8,9}$. To date, there are only a few predictions of the threedimensional (3D) TI in oxide systems ${ }^{10,11}$. Several iridium oxides suggested as TI candidates are still under debate because of the competition with the magnetic instability which is driven by electron-electron interactions and leads to novel phases other than TI such as Weyl semi-metal, axion insulator, and so on ${ }^{12,13}$. The competition among the small energy scales makes those iridates more interesting but less appropriate for practical applications especially in TI-based devices.

Here, we predict $\mathrm{YBiO}_{3}$ to be a new TI candidate, taking a first step toward bridging oxide electronics and TI. The cubic perovskite structure of $\mathrm{YBiO}_{3}$ makes it even more attractive. From the applications point of view, $\mathrm{YBiO}_{3}$ is of great advantage in several aspects: it has a large enough bulk band gap operative at room temperature. Moreover, it showed a high resistivity reported as the order of several $M \Omega \mathrm{m}^{14}$, which is hardly achievable in the $\mathrm{Bi}_{2} \mathrm{Se}_{3}$-family, the most prevailing 3D TI compounds. The highly resistive insulating phase will greatly help to reveal the fundamental properties of 3D TI through transport experiments by discerning the surface degrees of freedom from bulk. Furthermore, $\mathrm{YBiO}_{3}$ was originally deposited on top of a $\mathrm{LaAlO}_{3}$ substrate, and was used as a buffer layer for the high-temperature superconductor $\mathrm{YBa}_{2} \mathrm{Cu}_{3} \mathrm{O}_{7-\delta}$, which acts as a structural template for the epitaxial deposition of the superconductor and a diffusion barrier ${ }^{15}$. It implies the inborn character of $\mathrm{YBiO}_{3}$ to form interfaces with normal band insulators or superconductors. Considering the state-of-the-art synthesis techniques used in the artificial heterostrucutre of oxide perovskites, $\mathrm{YBiO}_{3}$ might possess great potential to show a rich variety of interface structures with other quantum phases. Probably, the TI phase in an oxide perovskite structure with a large band gap and high resistivity can provide an ideal platform to realize many exotic phenomena emerging at the interfaces, such as topologically protected surface states, giant magnetoelectric effects, Majorana Fermions, and so on ${ }^{16-19}$.

\section{Results}

Basic electronic structure. As a starting point, the basic electronic structure of $\mathrm{YBiO}_{3}$ without SOC is investigated in Fig. 1 where the orbital projections at each $k$-point are also depicted. It shows a semi-metallic phase with small 


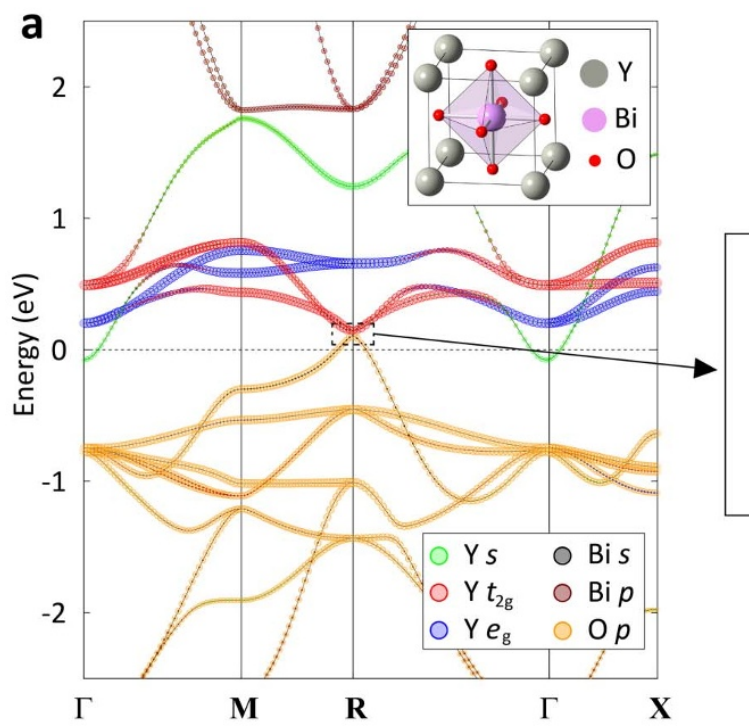

b

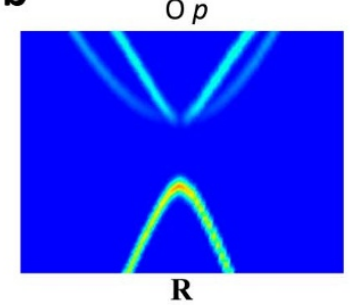

d

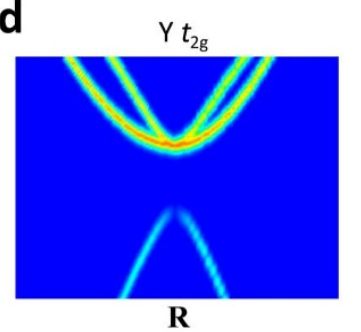

C

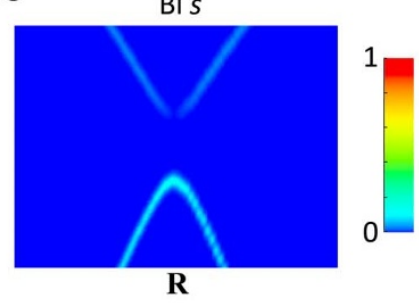

e

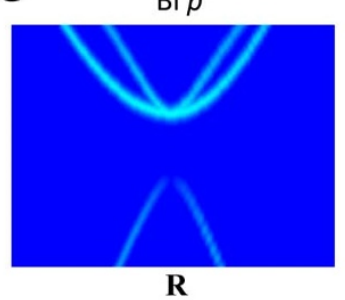

Figure $1 \mid$ Basic electronic structure of $\mathrm{YBiO}_{3}$. (a) The electronic band structure of $\mathrm{YBiO}_{3}$ without SOC. The circles at each $k$-point denote the atomic orbital projection of the Bloch wave-functions. The blowups of the low energy spectrum at around the $\mathrm{R}$ point are shown with the orbital contributions highlighted in colors for (b) O $2 p$, (c) Bi $6 s$, (d) Y $4 d t_{2 g}$, and (e) Bi $6 p$ orbital components, respectively.

electron and hole pockets around the $\Gamma$ and $\mathrm{R}$ points in the Brillouin zone, respectively. The valence bands near the Fermi level are mostly composed of oxygen $p$ states, as is usual in conventional oxide perovskites. More precisely, the highest valence band making the hole pocket around the R point has $s$ orbital symmetry originating from the hybridization between $\mathrm{Bi} 6 s$ and $\mathrm{O} 2 p$ orbitals (Fig. 1b, c). This $s$-like singlet, also found in halide perovskite compound $s^{20}$, is designated as the $|l=0\rangle \otimes|s=1 / 2\rangle$ state where $l$ and $s$ are the orbital and spin angular momentum quantum numbers, respectively; in other words, orbital degrees of freedom are quenched. On the other hand, the conduction bands close to the Fermi level consist of Y $5 s$ and $4 d$ orbital components. The Y $5 s$ band has a wide bandwidth forming an electron pocket at the $\Gamma$ point; the Y $4 d$ bands are narrow and split into the two-fold $e_{\mathrm{g}}$ and three-fold $t_{2 \mathrm{~g}}$ levels under the cubic symmetry. Considering the large distance between yttrium and the surrounding twelve oxygen atoms, the cubic crystal field splitting at the $\mathrm{Y}$ site is small and opposite to that in the octahedron center. Hence the $e_{\mathrm{g}}$ states are lower than $t_{2 \mathrm{~g}}$ at the $\Gamma$ point by $0.29 \mathrm{eV}$. But due to the hybridization between $\mathrm{Y} t_{2 \mathrm{~g}}$ and Bi $6 p$ states and the opposite sign of hopping parameters between $t_{2 \mathrm{~g}}$ and $e_{\mathrm{g}}$, the $t_{2 \mathrm{~g}}$ bands are located below the $e_{\mathrm{g}}$ bands at the $\mathrm{R}$ point. As a result, when SOC is excluded, the $t_{2 \mathrm{~g}}$ triplets and $s$-like singlet construct the low energy spectrum at around the R point, and become the main ingredients to determine the topological phase of this system. The $t_{2 \mathrm{~g}}$ triplets are the only SOC active bands, and they can be effectively mapped into $l_{\text {eff }}=1$ angular momentum states $\left(\mid l_{\text {eff }}\right.$ $=1\rangle \otimes|s=1 / 2\rangle)$ which are further split into $j_{\mathrm{eff}}=1 / 2$ and $j_{\mathrm{eff}}=3 / 2$ states by SOC, where $l_{\text {eff }}$ and $j_{\text {eff }}$ are the effective orbital and total angular momentum quantum numbers explained in detail later.

It is important to clarify the parities of the basis functions in determining the topological character of the inversion symmetric systems $^{21}$. Because the inversion is the symmetric operation at the $\mathrm{R}$ point, the parity eigenvalue is a good quantum number; in other words, there is no mixing between two eigenfunctions with opposite parities at the $\mathrm{R}$ point. Therefore, the wavefunction mixing at the $\mathrm{R}$ point in Fig. 1b-e clearly shows that the upper $t_{2 \mathrm{~g}}$ triplets and the lower $s$-like singlet have opposite parities. More specifically, the $\mathrm{Y} t_{2 \mathrm{~g}}$ orbitals couple to $\mathrm{Bi} 6 p$ states whereas the $\mathrm{O} 2 p$ orbital couples to $\mathrm{Bi}$ $6 s$. Because $\mathrm{Bi} 6 p$ and $6 s$ have odd and even parities with respect to the Bi center, respectively, the $t_{2 \mathrm{~g}}$ triplets are odd and the $s$-like singlet is even.
Non-trivial topological phase. In an attempt to elucidate the topological phase of $\mathrm{YBiO}_{3}$, the evolution of its electronic quasiparticle spectrum with varying SOC up to its full strength was scrutinized. The topological phase of the system can be verified by examining the topological phase transition during this process ${ }^{22}$. The variation of the SOC strength is designated as the ratio $x$ between the SOC value used in the calculations and in the real system. Figures 2a-d show the 3D plots of the electronic band dispersions on the $\Gamma-\mathrm{X}-\mathrm{R}-\mathrm{M}$ plane of the Brillouin zone as the SOC ratio $x$ gradually grows. Without SOC, the band topology is identified as the topologically trivial semi-metallic phase (Fig. 2a). When the SOC strength is slightly turned on, triply degenerate $t_{2 \mathrm{~g}}$ bands, the only SOC active ones, split into the lower $j_{\text {eff }}=1 / 2$ and the higher $j_{\text {eff }}=3 /$ 2 states, resulting in the $j_{\text {eff }}=1 / 2$ and $s$-like singlets closer at the $\mathrm{R}$ point. When $x$ reaches 0.213 , as shown in Fig. $2 \mathrm{~b}$, the bottom of the $j_{\text {eff }}=1 / 2$ band touches the top of the $s$-like band, forming a gapless Dirac cone. And by increasing $x$ a little bit larger, the Dirac cone acquires a mass gap again as the two singlet bands are inverted. The emergence of the gapless Dirac cone during the increment of $x$ directly indicates the topological phase transition from the trivial to the non-trivial phase. Due to the preformed electron and hole pockets, the trivial semi-metal is transformed into the topological semi-metallic phase. After the transition point, the non-trivial gap at the $\mathrm{R}$ point keeps rising in accordance with the increase of $x$, getting the volume of the hole pocket smaller and successively opening a real insulating gap. At $x=0.51$, the hole pocket shrinks to the point at $\mathrm{R}$, and so does the electron pocket at $\Gamma$ (Fig. 2c). When $x$ exceeds 0.51 , the topological semi-metal undergoes a transition to the topological insulating phase. Finally, with the full strength of SOC in Fig. 2d, $\mathrm{YBiO}_{3}$ manifests itself as a $3 \mathrm{D}$ TI with an indirect band gap of 0.183 $\mathrm{eV}$ between the conduction band minimum at $\Gamma$ and the valence band maximum at $(\pi, \pi, 0.58 \pi)$.

$\mathrm{YBiO}_{3}$ has an interesting feature in its indirect band gap; the conduction and valence band edges have different topological character. The conduction band bottom at $\Gamma$ mainly consists of the Y $5 s$ component, which is not influenced by SOC and does not participate in determining the band topology. On the other hand, the valence band top, the lower part of the non-trivial gap, has the $j_{\text {eff }}=1 / 2$ character split-off from the $\mathrm{Y} t_{2 \mathrm{~g}}$ bands by SOC, thus governing the topological phase of the system. The upper part of the non-trivial gap, the s-like singlet, is SOC inactive, and remains higher than the 
a

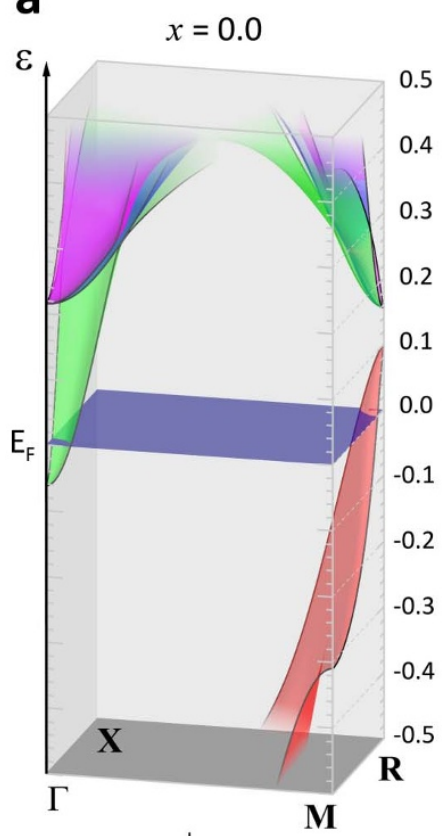

b

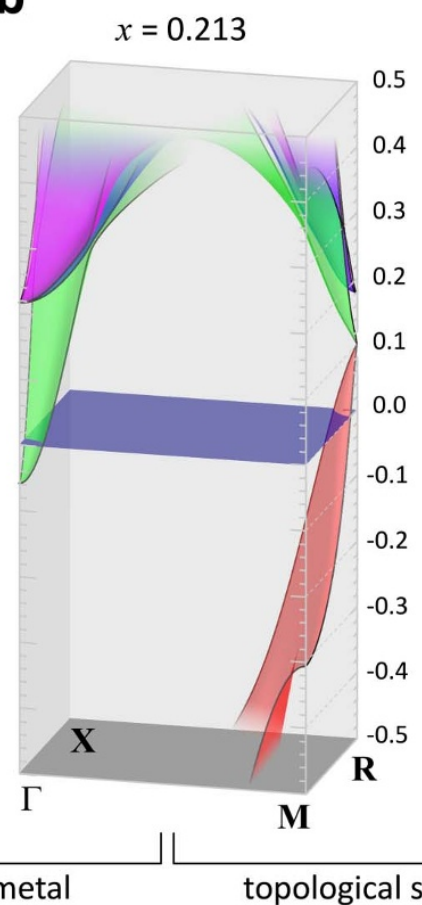

C<smiles>[124IH]</smiles>

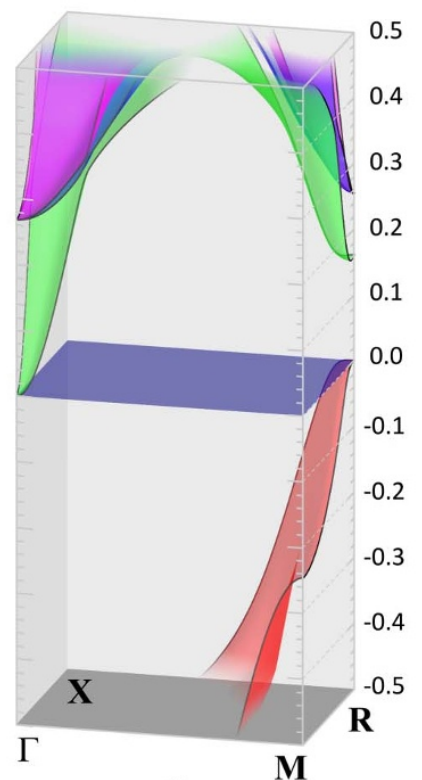

d

$x=1.0$
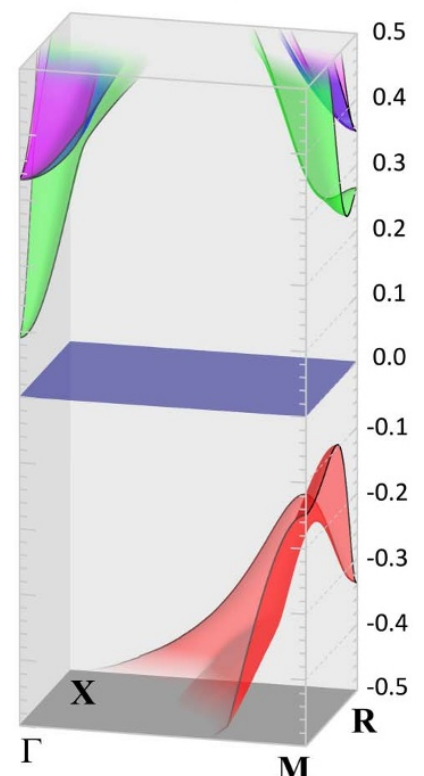

trivial semi-metal

topological semi-metal

topological insulator

Figure $2 \mid$ Evolution of the electronic structure with varying SOC. The 3D plots of the band structure on the $\Gamma$-X-R-M plane of the Brillouin zone are shown as the SOC ratio $x$ grows: (a) $x=0.0$, (b) $x=0.213$, (c) $x=0.51$, and (d) $x=1.0$. The dark blue planes in (a) $-(\mathrm{d})$ indicate the Fermi level.

conduction band minimum. The non-trivial gap is $0.33 \mathrm{eV}$ around the $\mathrm{R}$ point, which is larger than the real band gap. Once a $2 \mathrm{D}$ surface is introduced, topologically protected surface states are expected to reside inside the non-trivial gap, connecting the inverted bands.

Bulk-boundary correspondence. According to the bulk-boundary correspondence ${ }^{23,24}$, the topologically protected gapless modes should emerge at the boundary between two different topological phases.
The 2D surface of the 3D TI is one of the easily accessible phase boundaries between TI and vacuum, where such gapless states exist. The electronic structures of three conventional surface configurations in cubic perovskite $\mathrm{YBiO}_{3},(001),(110)$, and (111) surfaces, are depicted in Fig. 3. The slab geometries composed of 35 layers were constructed, whose microscopic energy scales related to the electronic degrees of freedom were obtained from the maximally localized Wannier function approach ${ }^{25,26}$. The topologically protected surface a

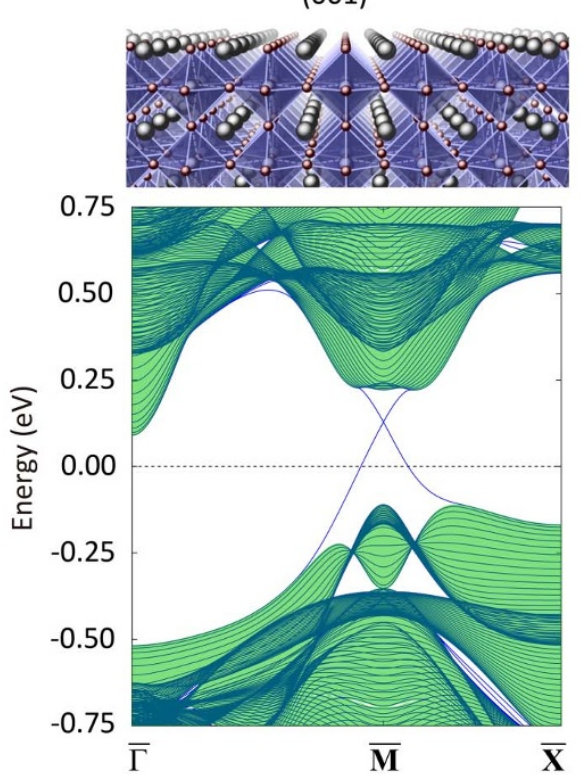

b
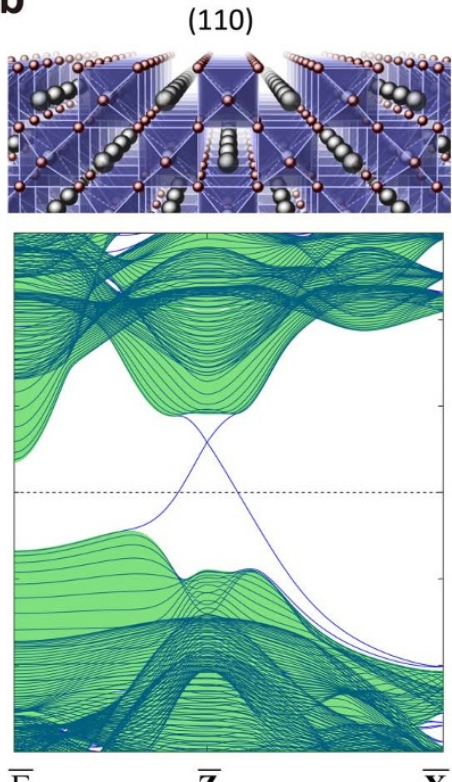

C
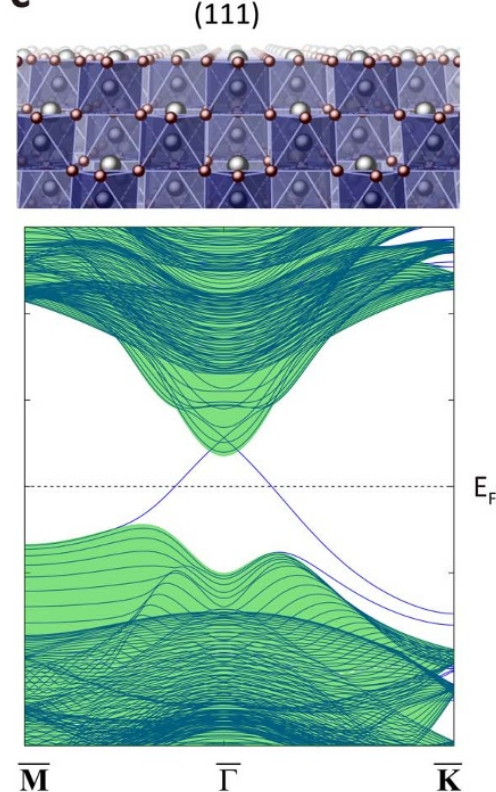

Figure 3 Topologically protected surface states. The surface geometries and the corresponding electronic band structures containing the topologically protected surface states for (a) (001), (b) (110), and (c) (111) surface configurations of the cubic perovskite $\mathrm{YBiO}_{3}$. The green shaded areas represent the surface-projected bulk continuum bands. 
states are shown inside the bulk gap region for all three surface configurations, which connect the inverted conduction and valence bands, present the single 2D Dirac cone, and reflect the 3D TI phase of the bulk $\mathrm{YBiO}_{3}$. Apart from the existence of the conducting surface states, details of the surface states are diverse. In case of the (111) surface, the Dirac point of the surface Dirac cone is embedded in the conduction band continuum, whereas the surface Dirac points are isolated from the bulk continuum and appeared inside the band gap in the (001) and (110) surface configurations. The difference originates from the different topological character of the conduction band minimum and valence band maximum, and the projection from the bulk to the surface Brillouin zone. More specifically, the $\Gamma$ and $\mathrm{R}$ points of the cubic Brillouin zone project into the same $\bar{\Gamma}$ point of the $2 \mathrm{D}$ hexagonal Brillouin zone in the (111) surface, and consequently the trivial conduction band edge and the non-trivial gap overlap as shown in Fig. 3c.

\section{Discussion}

All the above evidence say in unison that $\mathrm{YBiO}_{3}$ belongs to 3D TI via inverting the band character between the $j_{\text {eff }}=1 / 2$ state split-off from $\mathrm{Y} t_{2 \mathrm{~g}}$ orbital and the $s$-like state at the $\mathrm{R}$ point. However, there remains an unanswered question on what is the driving force for the nontrivial phase of $\mathrm{YBiO}_{3}$. The strong candidate is SOC only within the $t_{2 \mathrm{~g}}$ orbital of $\mathrm{Y} 4 \mathrm{~d}$, but it is inappropriate to explain the topological phase transition appearing in Fig. $2 \mathbf{b}$ because of the following two reasons. The SOC strength of Y $4 d$ is relatively small to induce the non-trivial phase. And more importantly, the $l_{\mathrm{eff}}=1$ triplet of the $t_{2 \mathrm{~g}}$ manifold is known to split into the higher $j_{\mathrm{eff}}=$ $1 / 2$ singlet and the lower $j_{\text {eff }}=3 / 2$ doublet by SOC; the sign of the SOC strength is effectively opposite to that of the original $l=1$ manifold ( $p$-orbital) ${ }^{27,28}$. The effective orbital angular momentum operator $\left(\mathrm{L}_{\mathrm{eff}}\right)$ is defined within the $t_{2 \mathrm{~g}}$ subspace in connection with the original angular momentum operator $(\mathrm{L})$ by

$$
\mathcal{P}_{t_{2 \mathrm{~g}}} \mathbf{L} \mathcal{P}_{t_{2 \mathrm{~g}}}=-\mathbf{L}_{\mathrm{eff}}
$$

where $\mathcal{P}_{t_{2 \mathrm{~g}}}$ is the projection operator to the $t_{2 \mathrm{~g}}$ manifold. Accordingly, $\mathrm{J}_{\text {eff }}=\mathrm{L}_{\text {eff }}+\mathrm{S}$ is the effective total angular momentum operator, and the effective SOC term is written as

$$
\mathcal{H}_{\mathrm{eff}}^{\mathrm{SOC}}=\mathcal{P}_{t_{2 \mathrm{~g}}}(\lambda \mathbf{L} \cdot \mathbf{S}) \mathcal{P}_{t_{2 \mathrm{~g}}}=-\lambda \mathbf{L}_{\mathrm{eff}} \cdot \mathbf{S}=\left\{\begin{array}{cc}
\lambda & \left(j_{\mathrm{eff}}=1 / 2\right) \\
-\lambda / 2 & \left(j_{\mathrm{eff}}=3 / 2\right)
\end{array}\right.
$$

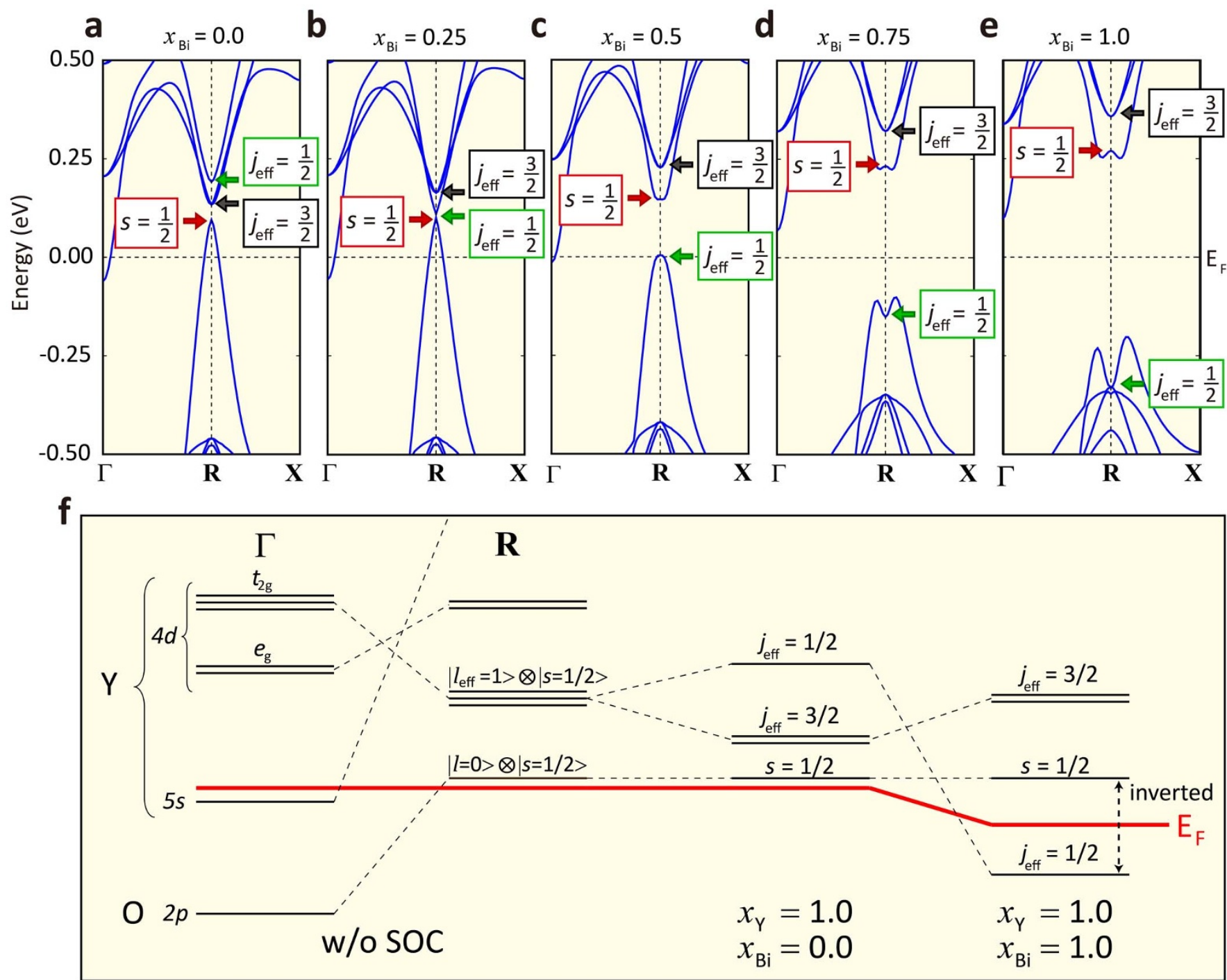

Figure $4 \mid \mathrm{SOC}$ of the $\mathrm{Bi} 6 p$ orbital. The bulk band structure of $\mathrm{YBiO}_{3}$ with varying $x_{\mathrm{Bi}}$, the $\mathrm{SOC}$ ratio of the $\mathrm{Bi}$ atom, with $x_{\mathrm{Bi}}=(\mathrm{a}) 0.0$, (b) 0.25 , (c) 0.5 , (d) 0.75 , and (e) 1.0 . The red, green, and grey arrows correspond to $s=1 / 2, j_{\text {eff }}=1 / 2$ singlets, and $j_{\text {eff }}=3 / 2$ doublet, respectively. The SOC values of other elements are maintained with their full strength. (f) The schematic diagram shows the evolution of the low energy spectrum as a function of the SOC strength of each atom, describing the topological phase of $\mathrm{YBiO}_{3}$. 
Therefore, SOC only in the Y $t_{2 \mathrm{~g}}$ orbital tends to form the $j_{\text {eff }}=3 / 2$ and $s=1 / 2$ states as the low energy spectrum near the R point rather than the $j_{\text {eff }}=1 / 2$ and $s=1 / 2$ singlets, which is inconsistent with the current situation.

In order to solve the contradiction, we vary the SOC strength of the $\mathrm{Bi}$ atom $\left(\lambda_{\mathrm{Bi}}\right)$ only, while maintaining the full SOC strength of other elements, envisaging the largest SOC of Bi $6 p$ states to play a crucial role. When we turn off $\lambda_{\mathrm{Bi}}$, the Y $t_{2 \mathrm{~g}}$ bands split into the lower $j_{\text {eff }}=$ $3 / 2$ doublet and the higher $j_{\text {eff }}=1 / 2$ singlet by $50 \mathrm{meV}$ at the $\mathrm{R}$ point (Fig. 4a), which agrees well with the previous description, and indicates the bare possibility of the topological insulating phase by SOC solely in $\mathrm{Y} 4 d$. As $\lambda_{\mathrm{Bi}}$ increases, the splitting between the $j_{\mathrm{eff}}=1 / 2$ and $j_{\text {eff }}=3 / 2$ states turns out to be reversed (Fig. $4 \mathbf{b}-\mathrm{e}$ ); $\lambda_{\mathrm{Bi}}$ substantially makes the $j_{\text {eff }}=1 / 2$ and $s=1 / 2$ singlets as constituents of the low energy spectrum, and finally induces an inversion between them, changing the band topology from trivial to non-trivial. Under the influence of the Bi $6 p$ orbital, the Y $t_{2 \mathrm{~g}}$ states follow the SOC splitting of the original $l=1$ states. All the behaviors of the Y $4 d t_{2 \mathrm{~g}}$ orbital states related to the topological phase of $\mathrm{YBiO}_{3}$ are driven by the SOC of the $\mathrm{Bi} 6 p$ orbital states through the strong $d$ - $p$ hybridization.

$\mathrm{YBiO}_{3}$, used as a buffer layer for the cuprate superconductor, is suggested as a new TI candidate. Combining the present theoretical results with the previous experimental facts and the future potentiality, its finding has more meaning than merely adding one more material in the TI catalogue. $\mathrm{YBiO}_{3}$ can provide a new platform for TI applications in terms of the large band gap, high resistivity, and an oxide perovskite material. The high residual bulk carrier density of conventional 3D TIs, although being improved ${ }^{29,30}$, mostly hampers the practical use of TI, and fails to meet the original goal of TI, insulating bulk and conducting surface. This material with its high bulk resistivity, which may stem from the chemical and structural integrity of oxide systems, can achieve the original goal. It is presumably useful for room temperature applications, feasible for the utilization of advanced fabrication techniques, and accessible for the various interface geometries with other quantum phases. These features are essential requirements for making better TI-based devices, and convincingly entitle $\mathrm{YBiO}_{3}$ as a promising TI material that assumes importance for future development.

\section{Methods}

First-principles calculations. To investigate the electronic structure and topological phase of $\mathrm{YBiO}_{3}$, first-principles calculations were performed using the full-potential linearized augmented plane wave (FLAPW) method ${ }^{31}$ with the generalized gradient approximation for the exchange-correlation functional. For the momentum space integrations, a $10 \times 10 \times 10$ mesh of special $k$-points was used in the $3 \mathrm{D}$ irreducible Brillouin zone wedge. We used a wave-vector cutoff of the basis set equal to $K_{\max }=$ 3.8 a.u. and an angular momentum expansion up to $l_{\max }=8$ for both the potential and charge density. The muffin-tin radii of $\mathrm{Y}, \mathrm{Bi}$, and $\mathrm{O}$ were $2.8,2.9$, and 1.8 a.u., respectively. The relativistic SOC was included self-consistently by a second variational procedure. The SOC contribution to the Hamiltonian is decomposed to that from atomic spheres and interstitial following the standard way in the LAPW method. When the SOC strength was adjusted, the spherical part of the SOC interaction of each sphere is multiplied by a simple constant $x$. A maximally localized Wannier function approach ${ }^{25,26}$ was employed for extracting the electronic energy scales and constructing the tight-binding Hamiltonian of the slab geometries. The projectors, as an aid to describing the low energy spectrum properly, consist of $\mathrm{Bi} p, \mathrm{O}$ $p$, and $Y s, p, d$ orbitals. The calculations were carried out with the experimental cubic lattice constant, $5.428 \AA^{15}$. Even though we adopted the simple cubic perovskite structure which might be feasible in the real growth condition, the structural properties of this compound need a further investigation.

1. Tokura, Y. \& Hwang, H. Y. Condensed-matter physics: Complex oxides on fire. Nat. Mater. 7, 694-695 (2008).

2. Herber, J. Materials science: Enter the oxides. Nature 459, 28-30 (2009).

3. Imada, M., Fujimori, A. \& Tokura, Y. Metal-insulator transitions. Rev. Mod. Phys. 70, 1039-1263 (1998).

4. Mannhart, J. \& Schlom, D. G. Oxide interfaces-An opportunity for electronics. Science 327, 1607-1611 (2010).

5. Hwang, H. Y. et al. Emergent phenomena at oxide interfaces. Nat. Mater. 11, 103-113 (2012).
6. Hasan, M. Z. \& Kane, C. L. Colloquium: Topological insulators. Rev. Mod. Phys. 82, 3045-3067 (2010).

7. Qi, X.-L. \& Zhang, S.-C. Topological insulators and superconductors. Rev. Mod. Phys. 83, 1057-1110 (2011).

8. Xia, Y. et al. Observation of a large-gap topological-insulator class with a single Dirac cone on the surface. Nat. Phys. 5, 398-402 (2009).

9. Zhang, $\mathrm{H}$. et al. Topological insulators in $\mathrm{Bi}_{2} \mathrm{Se}_{3}, \mathrm{Bi}_{2} \mathrm{Te}_{3}$ and $\mathrm{Sb}_{2} \mathrm{Te}_{3}$ with a single Dirac cone on the surface. Nat. Phys. 5, 438-442 (2009).

10. Pesin, D. \& Balents, L. Mott physics and band topology in materials with strong spin-orbit interaction. Nat. Phys. 6, 376-381 (2010).

11. Yang, B.-J. \& Kim, Y. B. Topological insulators and metal-insulator transition in the pyrochlore iridates. Phys. Rev. B 82, 085111 (2010).

12. Wan, X., Turner, A. M., Vishwanath, A. \& Savrasov, S. Y. Topological semimetal and fermi-arc surface states in the electronic structure of pyrochlore iridates. Phys. Rev. B 83, 205101 (2011).

13. Go, A., Witczak-Krempa, W., Jeon, G. S., Park, K. \& Kim, Y. B. Correlation effects on 3D topological phases: From bulk to boundary. Phys. Rev. Lett. 109, 066401 (2012).

14. Zhao, Y. et al. Development of a new series of buffer layers for REBCO coated conductors. Physica C: Superconductivity 463-465, 574-579 (2007).

15. $\mathrm{Li}$, G. et al. Possible new single-buffer layers for $\mathrm{YBa}_{2} \mathrm{Cu}_{3} \mathrm{O}_{7-y}$ coated conductors prepared by chemical solution deposition. J. Mater. Res. 22, 2398-2403 (2007).

16. Song, J.-H., Jin, H. \& Freeman, A. J. Interfacial dirac cones from alternating topological invariant superlattice structures of $\mathrm{Bi}_{2} \mathrm{Se}_{3}$. Phys. Rev. Lett. 105, 096403 (2010).

17. Fu, L. \& Kane, C. L. Superconducting proximity effect and Majorana Fermions at the surface of a topological insulator. Phys. Rev. Lett. 100, 096407 (2008).

18. Linder, J., Tanaka, Y., Yokoyama, T., Sudbø, A. \& Nagaosa, N. Unconventional superconductivity on a topological insulator. Phys. Rev. Lett. 104, 067001 (2010)

19. Qi, X.-L., Li, R., Zang, J. \& Zhang, S.-C. Inducing a magnetic monopole with topological surface states. Science 323, 1184-1187 (2009).

20. Jin, H., Im, J. \& Freeman, A. J. Topological insulator phase in halide perovskite structures. Phys. Rev. B 86, 121102 (2012).

21. Fu, L. \& Kane, C. L. Topological insulators with inversion symmetry. Phys. Rev. B 75, 045302 (2007).

22. Jin, H., Song, J.-H., Freeman, A. J. \& Kanatzidis, M. G. Candidates for topological insulators: Pb-based chalcogenide series. Phys. Rev. B 83, 041202 (2011).

23. Hatsugai, Y. Chern number and edge states in the integer quantum Hall effect. Phys. Rev. Lett. 71, 3697-3700 (1993).

24. Kane, C. L. \& Mele, E. J. $Z_{2}$ topological order and the quantum spin Hall effect. Phys. Rev. Lett. 95, 146802 (2005).

25. Marzari, N. \& Vanderbilt, D. Maximally localized generalized Wannier functions for composite energy bands. Phys. Rev. B 56, 12847-12865 (1997).

26. Souza, I., Marzari, N. \& Vanderbilt, D. Maximally localized Wannier functions for entangled energy bands. Phys. Rev. B 65, 035109 (2001).

27. Kim, B. J. et al. Novel $J_{\text {eff }}=1 / 2$ Mott state induced by relativistic spin-orbit coupling in $\mathrm{Sr}_{2} \mathrm{IrO}_{4}$. Phys. Rev. Lett. 101, 076402 (2008).

28. Jin, H., Jeong, H., Ozaki, T. \& Yu, J. Anisotropic exchange interactions of spinorbit-integrated states in $\mathrm{Sr}_{2} \mathrm{IrO}_{4}$. Phys. Rev. B 80, 075112 (2009).

29. Kim, D. et al. Surface conduction of topological Dirac electrons in bulk insulating $\mathrm{Bi}_{2} \mathrm{Se}_{3}$. Nat. Phys. 8, 460-464 (2012).

30. Hong, S. S., Cha, J. J., Kong, D. \& Cui, Y. Ultra-low carrier concentration and surface-dominant transport in antimony-doped $\mathrm{Bi}_{2} \mathrm{Se}_{3}$ topological insulator nanoribbons. Nat. Commun. 3, 757 (2012).

31. Wimmer, E., Krakauer, H., Weinert, M. \& Freeman, A. J. Full-potential selfconsistent linearized-augmented-plane-wave method for calculating the electronic structure of molecules and surfaces: $\mathrm{O}_{2}$ molecule. Phys. Rev. B 24, 864-875 (1981).

\section{Acknowledgments}

Support from the U.S. DOE under Grant No.DE-FG02-88ER45372 is gratefully acknowledged.

\section{Author contributions}

H.J. conceived the idea and designed the overall research. S.H.R. and J.I. performed the calculations and J.I. prepared figures. H.J. and A.J.F. wrote the paper with helps from all other co-authors. All authors reviewed the manuscript.

\section{Additional information}

Competing financial interests: The authors declare no competing financial interests. License: This work is licensed under a Creative Commons Attribution-NonCommercial-NoDerivs 3.0 Unported License. To view a copy of this license, visit http://creativecommons.org/licenses/by-nc-nd/3.0/

How to cite this article: Jin, H., Rhim, S.H., Im, J. \& Freeman, A.J. Topological Oxide Insulator in Cubic Perovskite Structure. Sci. Rep. 3, 1651; DOI:10.1038/srep01651 (2013) 